\title{
Perspektive für Forschung und Praxis
}

Neben Stadtpolitik und -verwaltung sind im Sinne einer integrierten nachhaltigen Stadtentwicklung Wissenschaft, Zivilgesellschaft und Wirtschaft wichtige Akteure für die Umsetzung der SDGs. So besteht beispielsweise vonseiten der Wissenschaft Interesse daran, komplexe Zusammenhänge über Wirkungsketten zu analysieren, um Aussagen darüber treffen zu können, wie einzelne Ziele der SDGs sich wechselseitig beeinflussen (Koch et al. 2019). Diese Komplexität zu erfassen und in praxistaugliche Handlungsempfehlungen zu überführen, stellt eine Herausforderung dar, die im Rahmen inter- und transdisziplinärer Forschungskooperationen geleistet werden kann (vgl. Abschn. 4.1). Im Sinne eines Wissensaustauschs zwischen Wissenschaft und Praxis muss auch ermittelt werden, ob und wenn ja welche konkrete wissenschaftliche Unterstützung Kommunen in Bezug auf die SDGs benötigen.

Zivilgesellschaftliche Akteure wie z. B. NGOs aber auch einzelne Bürger*innen, spielen eine entscheidende Rolle bei der Umsetzung der SDGs in den Städten (vgl. Abschn. 3.4 und 3.5). So sind sie diejenigen, die in den Städten wohnen und arbeiten, sich tagtäglich in ihnen bewegen und den Wunsch nach einer hohen Lebensqualität haben. Darauf hat die Ausgestaltung der Stadtentwicklung einen wichtigen Einfluss. Daher ist eine Einbeziehung der Zivilgesellschaft bei der Entwicklung der Nachhaltigkeitsziele der Städte sowie bei der Ausgestaltung und Umsetzung konkreter Maßnahmen essentiell. Da diese Beteiligungsprozesse, die mit Anhörung, Diskussion und Aushandlung einhergehen, sehr zeit- und arbeitsintensiv sind, formulieren Kommunen zum Teil Bedarf nach einer Unterstützung durch externe Akteure, die aus der Wissenschaft oder auch aus anderen Bereichen wie beispielsweise Beratungsunternehmen kommen können (vgl. Krellenberg et al. 2019).

Für Wirtschaftsakteure in der Stadt nimmt die Bedeutung der SDGs und ihr Beitrag zu deren Umsetzung zu, je mehr sie in Prozesse der Stadtentwicklung 
integriert sind. Durch die Zunahme öffentlich-privater Partnerschaften (ÖPP oder englisch Public-private-Partnerships), z. B. im Wohnungsbau, bei der Wasser/Abwasserver- und -entsorgung, bei Verkehrsprojekten oder jüngst auch bei der kooperativen integrierten Quartiersentwicklung, stellen die Agenda 2030 und die SDGs auch für die Wirtschaft einen wichtigen Handlungsrahmen dar. Insbesondere die vielfältigen Smart City Ansätze, die im Zug der Digitalisierung in wachsendem Maße umgesetzt werden, leben von ÖPP. Dies macht deutlich wie wichtig es ist, die Zielentwicklungs- und Aushandlungsprozesse unter Einbeziehung aller Akteure durchzuführen.

\subsection{Transdisziplinäre Ansätze: Zusammenarbeit von Wissenschaft, Stadtverwaltungen, Zivilgesellschaft und Wirtschaft}

Die gemeinsame Arbeit von kommunalen Verwaltungen, Zivilgesellschaft, Wirtschaft und Wissenschaft zur Umsetzung der SDGs kann im Rahmen transdisziplinärer Forschungsansätze erfolgen. Die transdisziplinäre Forschung versteht sich dabei grundsätzlich als akteursorientierte Forschung. Das heißt, dass Praxisakteure substanziell an einem Forschungsprojekt beteiligt und nicht nur Gegenstand der Untersuchung sind. Dabei liegt dem transdisziplinären Forschen ein realweltlicher Ansatz zugrunde, also der Anspruch aktuelle Herausforderungen zu adressieren und Lösungen für die Praxis zu erarbeiten. Das gegenseitige Lernen der beteiligten Akteure und der Anspruch konkrete Lösungen zu entwickeln charakterisieren diese Forschungsrichtung, die z. B. durch Ansätze des Co-Designs oder der Co-Produktion erreicht werden können. Partizipation und die Generierung neuen Wissens vereinen dabei die transdisziplinäre und die transformative Forschung (vgl. Abschn. 4.2) (Defila und Di Giulio 2018).

\section{Co-Design und Co-Produktion}

Von Co-Design und Co-Produktion spricht man, wenn Praxisakteure nicht Objekt der Forschung sind, z. B. in ihrem Handeln analysiert oder über ihre Meinung befragt werden, sondern aktiv in den Prozess als Co-Forschende einbezogen werden (Defila und Di Giulio 2018). Im Sinne eines Co-Designs identifizieren Wissenschaftler*innen sowie Praxisakteure gemeinsam die zu adressierenden Herausforderungen und Problemlagen. Das führt im besten Fall dazu, dass die Anliegen und das Wissen aller Beteiligten im Sinne einer Co-Produktion von Wissen in die Erarbeitung von Lösungsansätzen einfließen und Transformationsprozesse angeschoben werden. Man spricht daher bei einer Co-Produktion auch von einer lernenden und forschenden Kooperation von wissenschaftlichen und außerwissenschaftlichen Akteuren (Jahn et al. 2012). Ein Co-Produktionsprozess lebt demnach von relevantem Wissen aus wissenschaftlichen und außerwissenschaftlichen Quellen (Lang et al. 2012). Dem liegt 
die Erkenntnis zugrunde, dass gesellschaftliche Herausforderungen nur durch diese Art des gemeinsamen Lernens und Forschens angemessen adressiert und gemeistert werden können (Schuck-Zöller et al. 2017).

Co-Designprozesse stellen eine wichtige Methode dar, um die Lücke zwischen wissenschaftlicher Theorie und praxisorientiertem Handeln zu schließen. Gerade für die Umsetzung der SDGs und der Agenda 2030 können derartige Prozesse dazu beitragen, die notwendigen Nachhaltigkeitstransformationen anzuschieben. Co-Design und Co-Produktion sind im Gesamtkontext verschiedener partizipativer, transdisziplinärer und transformativer Forschungsmethoden zu sehen (vgl. Abschn. 4.2).

In transdisziplinären Forschungsprojekten kann durch die gemeinsame Produktion von Wissen und das gegenseitige Lernen die Umsetzung der SDGs in den Kommunen unterstützt werden. Transdisziplinäre Forschungsansätze können genutzt werden, um gemeinsam mit allen beteiligten Akteuren Zielkonflikte zu identifizieren und den Aufbau von Monitoringsystemen und die Evaluierung des Umsetzungsstands der SDGs voranzutreiben. Auch der Prozess der Entwicklung und Umsetzung von SDG-Indikatoren und Leitlinien sowie die Erarbeitung strategischer/operativer Ziele und Maßnahmen kann so gemeinsam angegangen werden, wobei eine Einbeziehung der Zivilgesellschaft unbedingt erfolgen sollte (vgl. Garschagen et al. 2018). Von besonderem Interesse für die Wissenschaft ist es, die Art der Beteiligung und des Austausches zu gestalten und zu begleiten. Die Komplexität realweltlicher Problemlagen, die mit einer transdisziplinären Forschung adressiert werden, erfordert es zudem, dass die Wissenschaft interdisziplinär zusammenarbeitet.

Der Bedarf an transdisziplinärer Forschung bei der Umsetzung der SDGs konnte im Rahmen eines Co-Design-Prozesses mit Vertreter*innen aus Forschung und Praxis in Deutschland (Krellenberg et al. 2019) erhoben werden. Akteure aus der Stadtentwicklungspraxis wurden dezidiert gefragt, welche Unterstützung durch die Wissenschaft sie als hilfreich erachten. Wissenschaftliche Expertise wurde insbesondere für die folgenden Aspekte nachgefragt:

- Identifikation bzw. Analyse von Zielkonflikten

- Aufbau von Monitoringsystemen

- Mechanismen zur Evaluierung des Umsetzungsstands der SDGs

- Entwicklung von Indikatoren

- Entwicklung und Umsetzung von Leitlinien, strategischer/operativer Ziele und Maßnahmen

- Integration verschiedener Daten

- Steuerung des Gesamtprozesses 
Auch die Entwicklung von Beteiligungs- und Austauschprozessen, die mit einer externen Prozessbegleitung und der Steuerung komplexer Abstimmungsprozesse einhergehen, wird als Beitrag der Wissenschaft gesehen.

\subsection{Bedarf für transformative Forschung}

Im Kontext transdisziplinärer Forschungsansätze haben sich in den letzten Jahren solche Forschungsformate weiterentwickelt, die gesellschaftliche Veränderungsprozesse nicht nur untersuchen, sondern Transformationsprozesse hin zu einer nachhaltigen Entwicklung gezielt erwirken bzw. mitgestalten können (vgl. WBGU 2011; Lahsen und Turnhout 2021). Diese werden als transformative Forschungsansätze bezeichnet und können gerade bei der Umsetzung der SDGs auf kommunaler Ebene eine wichtige Rolle spielen.

Ein Format transformativer Forschung stellt der Ansatz des Reallabors dar. Hier werden Forschungs- und Praxisziele zusammen adressiert, indem neue Erkenntnisse und neues Wissen durch den Austausch der Wissenschaft mit nicht-wissenschaftlichen Akteuren generiert und zugleich Transformationsprozesse angeschoben werden (Defila und Di Giulio 2018). Reallabore stellen eine Form des partizipativen Forschens dar, die einer normativen Orientierung wie beispielsweise den SDGs folgt. In einem Reallabor wird gemeinsam „experimentiert“. Experimentieren heißt hier, unterschiedliche Lösungsansätze zu durchdenken, diese in einem iterativen Prozess kontinuierlich zu reflektieren und, soweit möglich, umzusetzen. Insbesondere durch das Experimentieren und Reflektieren findet ein intensiver Austausch zwischen den beteiligten Akteuren statt.

Für die Umsetzung der SDGs in Städten bietet das transformative Forschen in einem Reallabor die Möglichkeit, die mit der Agenda 2030 angestrebten Nachhaltigkeitstransformationen anzustoßen. Durch die kontinuierliche Reflexion über Prozesse und Ziele können dabei unerwünschte Folgen - wie zum Beispiel die Nichterreichung der SDGs oder konträr laufende Entwicklung zwischen den SDGs - rechtzeitig erkannt werden. Durch das beständige Aushandeln im Rahmen des Prozesses können im besten Fall Konflikte, die aus den zum Teil konträren Perspektiven und Interessen der Akteure entstehen, vermieden werden. Dennoch können Reallabore auch scheitern, wenn zum Beispiel keine Einigung auf eine gemeinsame Lösungsstrategie möglich ist. Ein solches Scheitern kann für die Stadtentwicklungspraxis mit einem Prestigeverlust oder ökonomischen Folgen einhergehen. Unter anderem aus diesem Grund stehen Stadtverwaltungen Reallaboren teilweise kritisch gegenüber. Aus der Forschungsperspektive lässt 
sich jedoch auch das Scheitern eines Reallabors als Erkenntnisgewinn einordnen, da sich daraus ggfs. übertragbares Handlungswissen für andere Reallabore generieren lässt. Dennoch sollte einem Scheitern entgegengewirkt werden. Eine offene Kommunikation über die Arbeit im Reallabor, den Mehrwert, die Grenzen, Zeithorizonte und Herausforderungen von Beginn an ist unerlässlich. Dazu gehört eine sehr vertrauensvolle Arbeit zwischen allen Beteiligten mit klar definierten Rollen und Aufgaben auf Augenhöhe (Beecroft et al. 2018).

Alle an einem Reallabor beteiligten Akteure sollten an Wissen und Erkenntnissen gewinnen und dazu befähigt werden, etablierte Prozesse und Kommunikationskulturen auch nach dem Ende eines Reallaborprozesses fortzusetzen (Empowerment), um so dem transformativen Anspruch der SDGs gerecht zu werden und gleichzeitig Veränderungsprozesse in der Kommune zu initiieren. Findet dies nicht statt, laufen Reallabore Gefahr, dass sich Vertreter*innen von Verwaltungen und der Praxis selbst als Versuchsperson sehen (Eckart et al. 2018).

\section{Reallabore}

Reallabore wurden in den letzten Jahren im Rahmen zahlreicher drittmittelfinanzierter Forschungsvorhaben umgesetzt und dabei von verschiedenen Ministerien finanziert. So förderte z. B. das Wirtschaftsministerium Baden-Württemberg mit den Förderlinien „Reallabore, BaWü-Labs“ und „Reallabore Stadt“ (MWK Baden-Wuerttemberg 2021) jeweils sieben Reallabore. Auch die Zukunftsstadtförderlinie des Bundesministeriums für Bildung und Forschung (BMBF) hat Reallabore unterstützt; darunter das Projekt „,Ein urbanes Reallabor für die lokale Umsetzung der Sustainable Development Goals“ in Lüneburg (Innovationsplattform Zukunftsstadt 2021), das explizit die Umsetzung der SDGs adressiert. Wichtige Ergebnisse sind vor allem im Rahmen der durchgeführten Begleitforschungen erwachsen. Durch die Erkenntnisse aus den Begleitforschungen können nun vermehrt strukturierte und praxisorientierte Grundlagen zur „,Reallaborforschung“ bereitgestellt werden. Es liegen Beschreibungen zu den eingesetzten Methoden vor, die für die Umsetzung konzeptioneller Reallabor-Ideen herangezogen werden können. Insbesondere aus den Erfahrungen derjenigen Reallabore, die als Treiber von Transformationen genutzt werden, kann Wissen über Methoden und Prozesse für den Einsatz zur Umsetzung der SDGs und zu Urbanen Nachhaltigkeitstransformation eingesetzt werden (Krellenberg und Koch 2021). Ein weiterer wichtiger Mehrwert aus den verschiedenen Reallaboren kann durch die Vernetzung von Akteuren entstehen. 
Open Access Dieses Kapitel wird unter der Creative Commons Namensnennung 4.0 International Lizenz (http://creativecommons.org/licenses/by/4.0/deed.de) veröffentlicht, welche die Nutzung, Vervielfältigung, Bearbeitung, Verbreitung und Wiedergabe in jeglichem Medium und Format erlaubt, sofern Sie den/die ursprünglichen Autor(en) und die Quelle ordnungsgemäß nennen, einen Link zur Creative Commons Lizenz beifügen und angeben, ob Änderungen vorgenommen wurden.

Die in diesem Kapitel enthaltenen Bilder und sonstiges Drittmaterial unterliegen ebenfalls der genannten Creative Commons Lizenz, sofern sich aus der Abbildungslegende nichts anderes ergibt. Sofern das betreffende Material nicht unter der genannten Creative Commons Lizenz steht und die betreffende Handlung nicht nach gesetzlichen Vorschriften erlaubt ist, ist für die oben aufgeführten Weiterverwendungen des Materials die Einwilligung des jeweiligen Rechteinhabers einzuholen. 